\title{
Some Procedures in Mitigating Conducted Electromagnetic Interference
}

\author{
M. I. Buzdugan, H. Bălan, T. I. Buzdugan \\ Technical University from Cluj-Napoca, 18, Memorandumului str., Cluj-Napoca (Romania) \\ E-mail: mircea.buzdugan@insta.utcluj.ro,horia.balan@eps.utcluj.ro,tudor.buzdugan@aut.utcluj.ro
}

\begin{abstract}
In our opinion, the problem of conducted interference generated by power converters, represents not a simply mater of harmonics or even interharmonics, as usually is considered, but really a broader issue, which requires a careful treatment of a continuous spectrum of frequency, extending until 30MHZ. The following paper presents a conducted electromagnetic interference problem in the case of a power converter, included in one kinetic therapy equipment (actually a treadmill).

Analysis is made, considering only conducted emissions at the mains supply port of the equipment. Tests carried out, outlined a few procedures in the analysis and mitigation of electromagnetic conducted interferences using electromagnetic interference mains filters.

Virtually all products contain a power supply filter as the last circuit that noise currents pass through before they exit the product through the power cord. Sometimes, properly designed transformers can provide inherent filtering, and so can obviate the need for an intentional filter. In other cases, like the one presented in the paper, mains filters are not enough for mitigating conducted interferences and they must be replaced, or simpler retrofitted by an additional one, the filters' cascade being able more often to mitigate perturbations. Mains passive EMI filters carry potentially high currents at dangerously high voltages, so the choice of these filters is an essential issue.

From the paper it can be seen that the EMI filter issues should be considered with careful attention by designers, because they are not only ancillary devices, as they are more often treated.
\end{abstract}

\section{Keywords}

Electromagnetic compatibility EMC, electromagnetic interference EMI, conducted emissions, EMI filter, common mode, differential mode.

\section{Introduction}

Technical literature is abounding in definitions for electromagnetic compatibility (EMC), but perhaps the most synthetic and eloquent one is that EMC consists is the absence of effects due to electromagnetic interference (EMI) [1].

Because electric and electronic systems penetrate more deeply into all aspects of society, both the potential for interference effects and the potential for serious EMIinduced incidents increase.
It is well known that the threat of EMI is controlled by adopting the practices of EMC, which include the intrasystem and inter-system EMC. Difficulty arises when intra-system meets inter-system, when the two approaches are confused one with the other, or at the interface where they meet.

Further the transfer of electromagnetic energy (with regard to the prevention of interference) is broken into four subgroups: radiated emissions, radiated susceptibility, conducted emissions, and conducted susceptibility.

There are basically two classes of EMC requirements that are imposed on electric and electronic systems; those mandated by governmental agencies and those imposed by the product manufacturer.

The legal requirements are imposed in order to minimize the interference produced by the product. However, compliance with these EMC requirements does not guarantee that the product will cause no interference. On the other hand, EMC requirements that manufacturers voluntarily impose on their products are intended to result in customer satisfaction (in order of reliable). Compliance with both of these EMC requirements is critical to the success and the good reputation of the product in the marketplace.

Regulatory agencies impose limits on these conducted emissions because they are placed on the utility power system net of the installation.

The utility power distribution system in an installation is a large array of wires connecting the various power outlets from which the other electronic systems in the installation receive their $\mathrm{AC}$ power. It therefore represents a large "antenna" system from which these conducted emissions can radiate quite efficiently, causing interference in the other electronic systems of the installation. Thus the conducted emissions may cause radiated emission, which may then cause interference. Ordinarily, the reduction of these conducted emissions is somewhat simpler than the reduction of radiated emissions since there is only one path for these emissions that needs to be controlled: the unit's power cord. However, it is important to realize that if a product fails to comply with the limits on conducted emissions, compliance with the limits on radiated emissions is a 
moot point. Therefore controlling conducted emissions of a product has equal priority with the control of radiated emissions [2].

For ease of measurement and analysis, in the commercial tests, radiated emissions are assumed to predominate above $30 \mathrm{MHz}$, while conducted emissions are assumed predominant below $30 \mathrm{MHz}$.

There is of course no magic changeover at $30 \mathrm{MHz}$. But typical cable lengths tend to resonate above $30 \mathrm{MHz}$, leading to anomalous conducted measurements, while measurements radiated fields below $30 \mathrm{MHz}$ will of necessity be made in the near field closer to the source giving results that do not necessarily correlate with real situations.

At higher frequencies, mains wiring becomes less efficient as a propagation medium, and the dominant propagation mode becomes radiation from the equipment or wiring in its immediate vicinity.

Perhaps the most important aspect of becoming effective at EMC design is to begin thinking of the nonideal behavior of electrical components in addition to the ideal behavior that we have been taught to keep in mind.

If we think only in terms of ideal behavior of electrical and electronic components, we will not be able to see or anticipate the nonideal electrical paths and hence will not be able to consider other possible causes for conducted or radiated emissions.

Therefore we will have inadvertently reduced our possibilities for correcting EMC problems; we will not have the ability to see a schematic beyond its appearance.

\section{Conducted Interferences in $\mathrm{AC}$ Variable Speed Drive}

In technical literature about power converters, the electromagnetic interference is analyzed more or less accurate, focusing especially on harmonics.

In general, the overall efficiency and performance of the electric motor is considered dependent on the quality of the current to the motor, so the AC converter is considered a major source of both conducted and radiated electromagnetic interference (EMI).

The two main areas of EMI generation involved are in general [3]:

- The supply side (mains), the harmonics generated by the rectifier fall into the frequency spectrum up to about $3 \mathrm{kHz}$ and are conducted back into the power system. The radiated EMI from the rectifier is of relatively low frequency (low di/dt).

- The motor side, due to the high inverter switching frequencies (typically between $2 \mathrm{kHz}$ to $20 \mathrm{kHz}$ ), high frequency harmonics up to $10 \mathrm{MHz}$ (RFI) are generated by the inverter and conducted along the cable to the motor. The EMI radiations from this cable are therefore of relatively high frequency, due to high values of $\mathrm{dv} / \mathrm{dt}$.

Supply side harmonic interference is a continuous distortion (up to $3 \mathrm{kHz}$ ) of the normal sinusoidal current waveform. The distortion frequencies are multiples of the fundamental $50 \mathrm{~Hz}$ frequency.

Motor side interference is a continuous high frequency distortion (above about $10 \mathrm{kHz}$ ) superimposed on top of the normal sinusoidal waveform.
AC converters do not themselves radiate a high level of EMI energy. The electromagnetic fields in the immediate vicinity $(<100 \mathrm{~mm})$ of the converter can be quite high, but these diminish quite quickly according to the inverse square law and are insignificant at a distance of about $300 \mathrm{~mm}$. When AC converters are mounted in metal enclosures, the electromagnetic radiation is largely eliminated. The main mechanism of propagation of the EMI is through the supply cables, the cables to the motor and most importantly through the earth connections. The supply cable is the most important route for the transfer of EMI.

Conduction along the control and communications cables is fairly rare because these cables are usually well shielded and their source impedance is high.

In our opinion, the problem is not such a simple one, but on a contrary it must be considered carefully, especially by manufactures, because far from being only a mater of harmonics or interharmonics, conducted interference represents a continuous broad spectrum of frequency, extending until 30MHZ.

Starting from this point, we have performed in the electromagnetic compatibility laboratory of our university, conducted emission studies, on a prototype of one kinetic therapy equipment (actually a treadmill).

The main part of the equipment consists in the single phase SYSDRIVE 3G3EV-AB015M-CUE, 1.5kW power converter (OMRON), controlled by pulse width modulation (PWM) in the range from OVDC until 10 VDC. The power converter is driving a $460 \mathrm{~W}$ three phase induction motor.

At the supply terminals is fitted a single phase VAC noise filter, recommended by the manufacture of the power converter (a PNF 2020-YN of 2A rated current, produced by SOSHIN ELECTRIC CO., LTD).

The equipment is controlled by a computer using a serial through interface USB. The block diagram of the treadmill equipment is depicted in Fig.1.

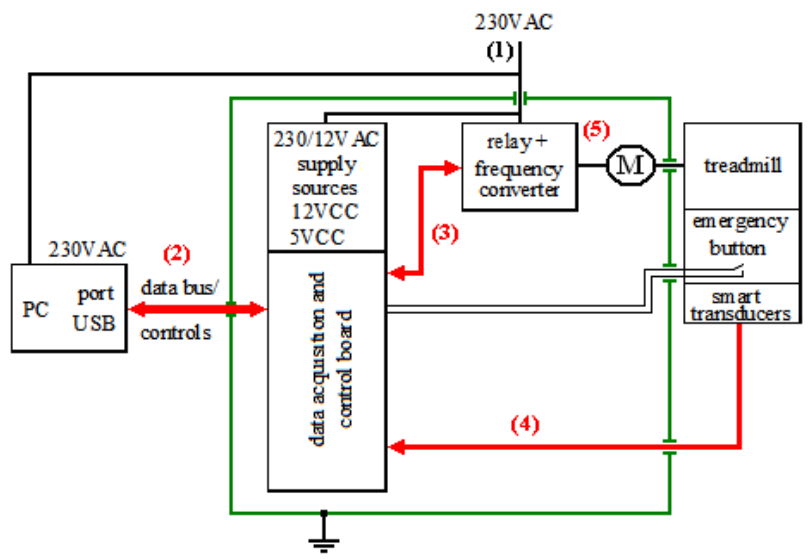

Fig. 1. Schematic diagram of the treadmill equipment

From the diagram, one may depict the main conducted EMI path inter-system $(1,2,4)$ and intra-system $(3,5)$. It is obvious that there are also secondary conducted EMI path and radiated emissions. We have focused mainly on the conducted electromagnetic interference at the 230VAC mains port, which often corrupted the firmware package of the equipment. This was a very annoying mater for the manufacturer of the prototype, because in 
the computer there are usually stored several medical recovery procedures and the data base containing the time evolution of the recovering patients.

The main purpose of the conducted emission tests consisted in evaluating noise currents that exit the product's AC power cord conductors (the emission path 1, depicted in Fig.1.).

In order to record conducted emissions at the mains supply voltage, we have used a line impedance stabilization network (LISN) and a spectrum analyzer (the schematic setup is presented in Fig. 2. and the experimental setup in Fig. 3.).

In this procedure, the device under test (DUT) is connected directly to an EMI filter, the power cord and the LISN.

Usually, the first objective of every LISN is to present constant impedance to the product's power cord outlet over the frequency range of the conducted emission test.

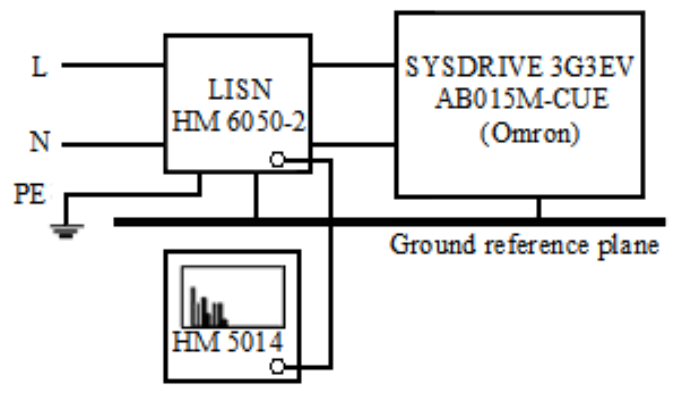

Fig. 2. The schematic setup

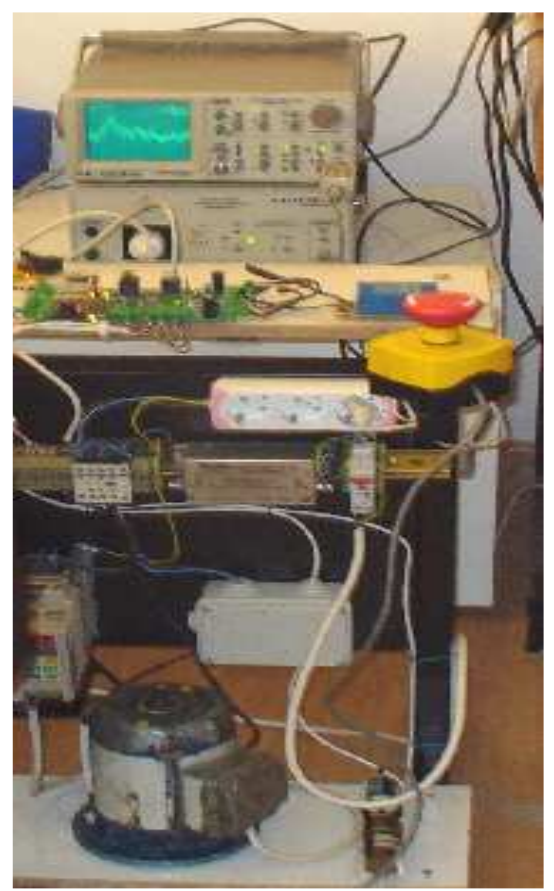

Fig. 3. The experimental setup

Inside the LISN the power lines are terminated with a well-defined impedance network, against each other and against ground, LISN being in principle a filter network. The two major objectives of the LISN are: to present constant impedance $(50 \mathrm{~V})$ between the phase conductor and the safety wire (the "green wire") and between the neutral conductor and the safety wire, and to prevent external conducted noise on the power system net from contaminating the measurement. These two objectives are to be satisfied only over the frequency range of the conducted emission test $(90 \mathrm{kHz}-30 \mathrm{MHz})$.

Another requirement for the LISN is to allow the $50 \mathrm{~Hz}$ $(60 \mathrm{~Hz})$ power required for the proper product's operation.

For measurements with a Spectrum Analyzer/EMC Receiver, the EMC signal is available after having passed a high pass filter.

Two identical networks provide the asymmetric noise emission signals of the DUT's power lines L1 and N. The user can choose between the signals, the selected one will be available at the test signal outlet.

The stabilization network (simulation for the AC power lines) is arranged in form of a "V".

When working with a Spectrum Analyzer/EMC Receiver it is also highly recommended to enable a Transient Limiter.

Basic spectrum analyzers are not an alternative to a measuring receiver in a full compliance set-up because of their limited sensitivity and dynamic range, and susceptibility to overload. However, the spectrum display is extremely valuable for confirming the frequencies and nature of offending emissions, as is the ability to narrowin on a small part of the spectrum.

When combined with a tracking generator, a spectrum analyzer is useful for checking the HF response of circuit networks.

As we have already presented in Fig. 2, we have used a HM 6050-2 LISN and a HM 5014 spectrum analyzer, both manufactured by HAMEG Instruments [5, 6].

The unique features of this spectrum analyzer are the extensive EMC measurement capabilities. These include the amplitude indication in Quasi Peak and Average modes. For the precise evaluation of the signals a marker is provided that will give the readout for amplitude and frequency on-screen.

An additional advantage is that newly acquired signals can be compared with the content of the reference storage.

Complicated and repeatedly used equipment adjustments can be saved by use of the Save/Recall function.

The analyzers are supplied with an RS-232 interface for PC communication and the firmware package SW5010.

The embedded tracking generator is a special signal source whose RF output frequency tracks (follows) some other signal beyond the tracking generator itself. In conjunction with the spectrum analyzer, the tracking generator produces a signal whose frequency precisely tracks the spectrum analyzer tuning.

Using the tracking generator/spectrum analyzer combination makes possible for several tests related to EMC measurements:

- characterize the loss of RF cables. Cable attenuation versus frequency must be accounted for in an overall emissions measurement;

- characterize components, filters, attenuators and amplifiers, which are vital for effective EMC remedies;

- determine structural and circuit resonances.

The RF noise injected level measured in the mains network revealed an electromagnetic interference exceeding the limit specified by the standard EN 55011 for conducted emissions [7], both between L-PE (Fig.4.) and N-PE (Fig.5), in logarithmic scale. 


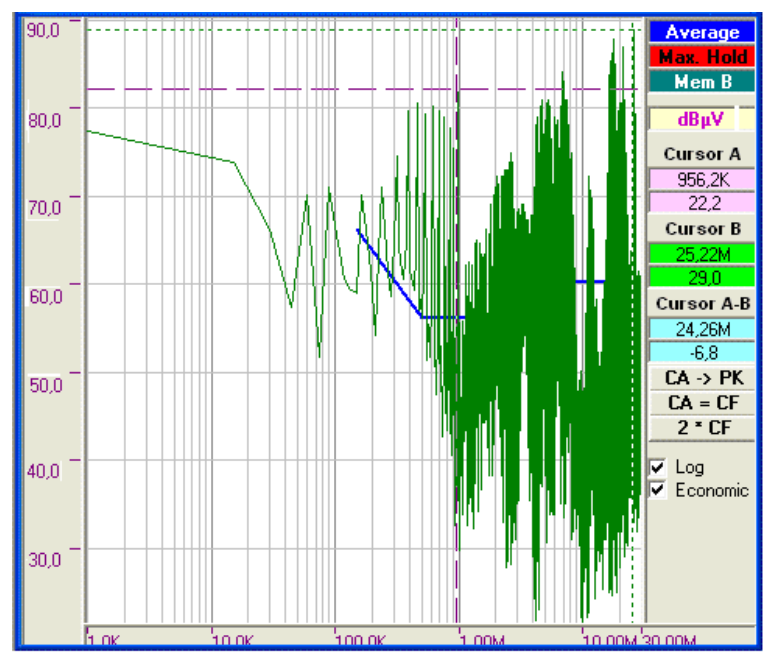

Fig. 4. Conducted emissions between L-PE

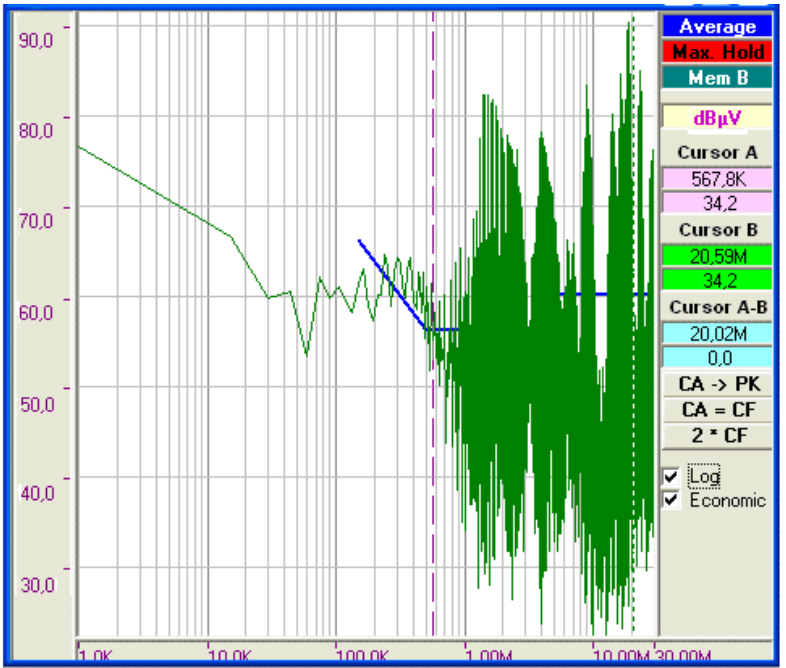

Fig. 5. Conducted emissions between N-PE

The conducted emissions are close to $90 \mathrm{~dB} \mu \mathrm{V}$ in both cases, compared to the average limits set by the emission standard EN 50011, depicted in Fig. 6. (average value = blue line and quasi peak value $=$ red line). The average limit set by the standard EN 50011 is $60 \mathrm{~dB} \mu \mathrm{V}$.

The limits of the standard differ by more or less with $10 \mathrm{~dB} \mu \mathrm{V}$ because the limits set on the spectrum analyzer due to calibration.

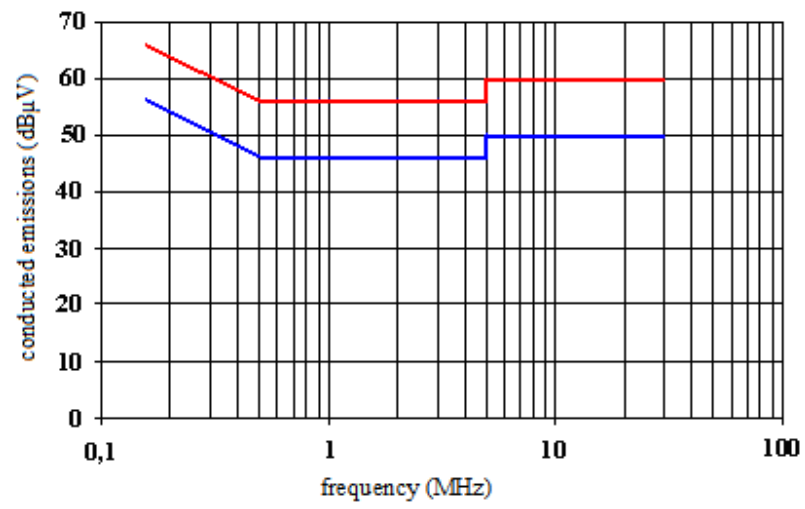

Fig. 6. Emission limits set by the standard EN 55011

The emission limits set by EN 55011 could be too high for normal use of equipments with a greater complexity, so they may create interference, like we have previously seen.

One of the common efficient countermeasures consists in mitigating conducted interferences at the mains supply, by fitting a proper designed passive EMI filter.

\section{Passive EMI Filters, efficiently mitigate conducted emissions}

Very often, engineers call passive EMI filters as black magic, because mainly four reasons: first, there has not been a well-defined design method, second, the input and output impedances are not constant over the band of interest and the impedances are usually o good guesses, at best, because the measurements are costly to make and are rarely repeatable, third, the filter insertion loss test method specifications often confuse or influence the design method and fourth, the design methods outlined in various publications are generally very complex. Most require measurements that are difficult to make and time consuming. Often expensive additional equipments are required to obtain the needed parameters [8].

Standard filters differ greatly from EMI filters. So, most of the energy in the stop band of the filter is reflected to its source. This fact is often overlooked in both standard filter and EMI technology. The remaining energy is expended in the inductors through the DC resistance of the coil, the core losses (eddy currents and hysterisis), and the equivalent series resistance of the capacitors. Standard filter design uses several excellent programs computers (Butterworth, elliptic, Chebyshev, and M derived, etc.) so the input and output impedances of the source and load (usually the same) is readily known, along with the allowed band-pass ripple, the 3-dB point, and the stop frequency.

Conversely, most EMI filter manufacturers design need only the low-pass filters (all pole networks) for the required EMI attenuation. They rarely build band pass or other conventional filters. The technology used in standard filters is truly different from that used in EMI filters. The EMI filter design is very loose compared with that used by the standard filter manufacturer. The EMI filter component values are very flexible, so the engineer can use standard values. These filters are adjusted only to meet the required insertion loss specification assuming the rest of the specification is met.

The languages spoken by the two groups are also different. The first one often speaks of poles, zeros, group delay, predistortion, attenuation, and terms such as the order of the filter. The EMI filter designer thinks in terms of attenuation, insertion loss, filter voltage drop, filter voltage rise, and the number of filter sections required to meet the insertion loss.

Mains EMI filters carry potentially high currents at dangerously high voltages, so care is essential in their choice. The operating voltage and the current rating of components can be decided once the specification is known. The basic specification should also include mechanical details such as the enclosure size, and the limit of weight. In addition the EMC performance and the allowable leakage current should be specified. 
The electrical specification must also comply with national safety standards.

Mains filters are tested with a $50 \Omega$ source and load impedance because most $\mathrm{RF}$ test equipments have a characteristic impedance of $50 \Omega$. This allows consistent test results and allows direct comparison between one design and another.

However, because the source and load impedance in practical situations are not generally $50 \Omega$, the attenuation predicted for a design based on this specification is generally optimistic compared with its performance in working equipment.

In the real applications, the source and load impedances, $\mathrm{Z}_{\mathrm{S}}$ and $\mathrm{Z}_{\mathrm{L}}$, are complex and in general unknown at the frequencies of interest for suppression [9]. If either or both has a substantial reactive component then resonances are created which may convert an insertion loss into an insertion gain at some frequencies. In the case of mains supplies, the source and load impedance varies widely with frequency.

The source impedance is variable over time and can be anywhere from $2 \Omega$ to $2000 \Omega$. The actual impedance is dependent on the loads that are connected to it and the frequency of interest. The characteristic impedance of the mains lead to the load is around $150 \Omega$, and the load itself may have variable impedance.

Differential mode impedances may be predictable if the components which make up the source and load are well characterized at RF, but common mode impedances such as are presented by cables or the stray reactance of mechanical structures are essentially unpredictable.

For the treadmill equipment analyzed above, the only mains filtering cell was the PNF 2020-YN, produced by SOSHIN ELECTRIC CO., LTD., (rated current 20A, rated voltage $250 \mathrm{VAC}$ and leakage current $1.5 \mathrm{~mA}$ ), whose schematic is depicted in Fig. 7., along with the frequency data is depicted in Fig 8. (recall that for the depicted frequency data of the PNF 2020-YN filter, the dotted line is the relevant one until $8 \mathrm{MHz}$ ), [10].

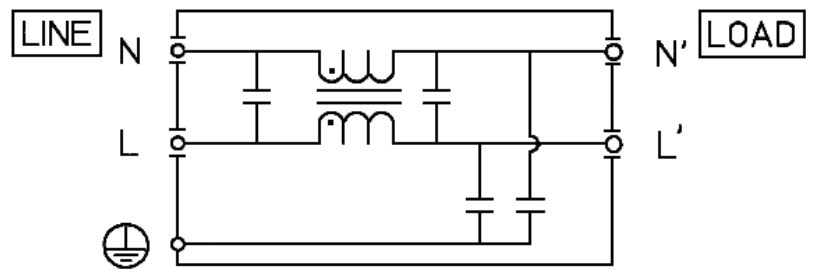

Fig. 7. Schematic of the PNF 2020-YN filter

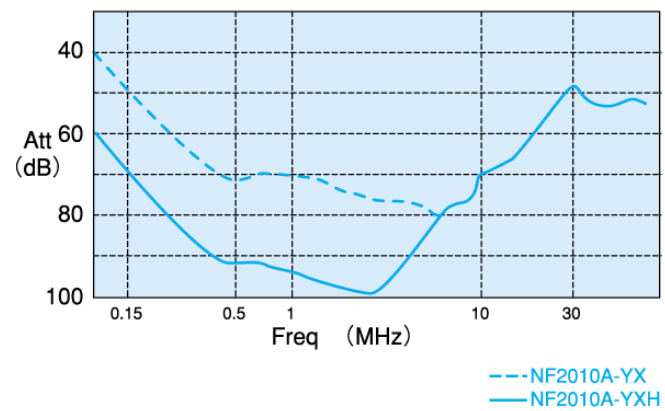

Fig. 8. Data frequency for the PNF 2020-YN filter

Theoretically, each component of EMI filter can mitigate either the differential mode or common mode emission.
EMI filters are generally composed by a common mode capacitor $(\mathrm{CY})$, a differential mode capacitor $(\mathrm{CX})$, a common mode choke (LCM) and a differential mode choke (LDM), the two least components being used to limit the common mode and differential mode emissions, respectively.

The ferrite core is used to be common mode choke and powder core is used to be differential mode choke because of low core loss, magnetic stability and high permeability at high frequency [11].

However, the size and weight of EMI filter principally depend on the size of choke. Therefore, there are many researches pointed on integrated common mode and differential mode choke.

Finally, the performance, the conducted interference and insertion loss, of common-mode chokes are compared and verified by the experiment.

In the schematic diagrams (Fig. 7.), one may easily observe that the filter is not fitted with a properly common mode choke (such as a Zorro inductor or a balun choke). This type of filter is mainly intended to mitigate differential emissions and not really common mode emissions [12].

But in electromagnetic conducted emissions, stopping the common mode perturbation should by compulsory in many situations. This was the reason that we decided to add an extra EMI filtering cell, properly fitted with a common mode balun choke (Fig.9.).

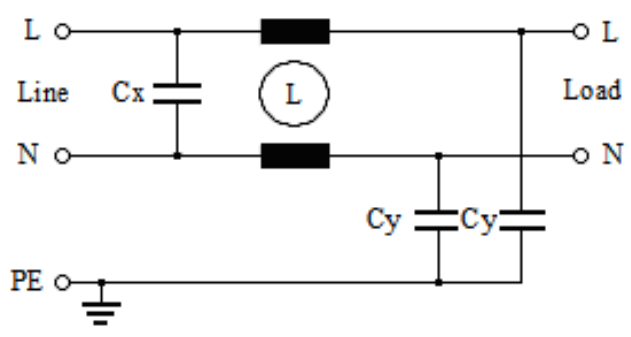

Fig. 9. Typical mains EMI filter

The typical ready-made EMI filter is a balanced $\Pi$ type, manufactured by Arcotronics Ltd. (F.AM.D.-3600.ZC.). Capacitors $\mathrm{C}_{\mathrm{Y}}$ attenuate common mode interference. The effectiveness of the $C_{Y}$ capacitors depends very much on the common mode source impedance of the equipment. The common mode choke $\mathrm{L}$ consists of two identical windings on a single high permeability toroidal core, configured so that differential (line-to-neutral) currents cancel each other. This allows high inductance values, typically $1-10 \mathrm{mH}$, in a small volume without fear of choke saturation caused by the mains frequency supply current, due to the high $A_{L}$ values [13].

The parameters of the filter are: rated voltage $=250 \mathrm{VAC}$, rated current $=16 \mathrm{~A}, \mathrm{C}_{\mathrm{X}}=0,6 \mu \mathrm{F}$ (X2 class), $\mathrm{C}_{\mathrm{Y}}=$ $2 \times 2500 \mathrm{pF}$ (Y2 class), $\mathrm{L}=2 \times 1 \mathrm{mH}$, leakage current $\mathrm{I}_{\mathrm{L}}=2 \times 0.23 \mathrm{~mA}$ and the temperature range $-25^{\circ} \mathrm{C}$ to $+85^{\circ} \mathrm{C}$. According to the catalog sheet, in the frequency range of interest the filter attenuation in common mode exceeds $50 \mathrm{~dB} \mu \mathrm{V}$.

We have analyzed once again the treadmill equipment, this time after retrofitting the cascade F.AM.D.-3600.ZC plus PNF 2020-YN.

The new results are depicted in Fig. 10. and Fig. 11. 


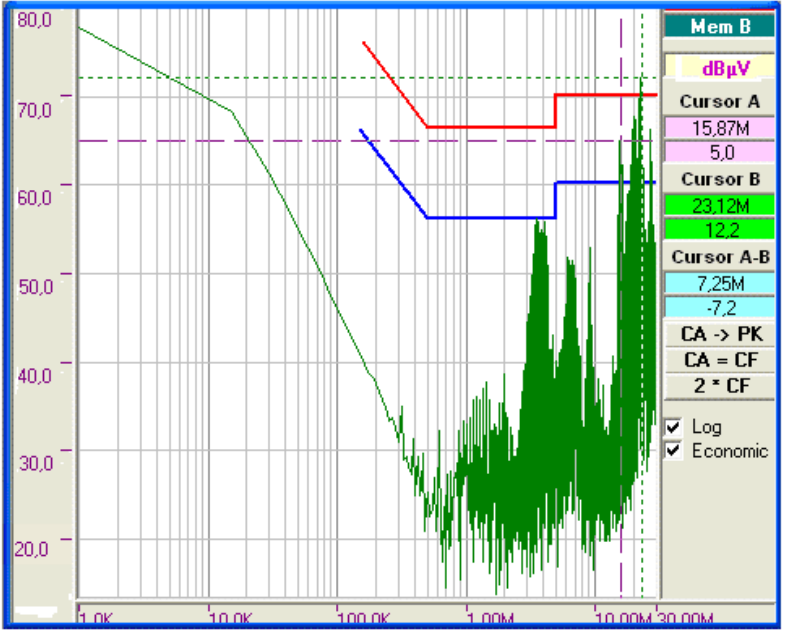

Fig. 10. Conducted emissions between L-PE

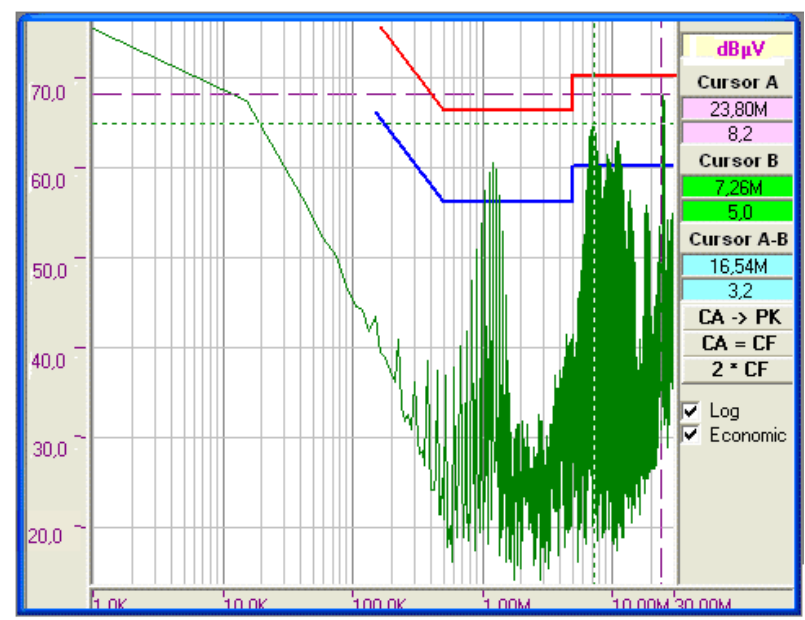

Fig. 11. Conducted emissions between N-PE

The results of the conducted emissions revealed that the cascade cells of EMI filters represents a very effective solution in stopping conducted emissions, because they dropped by almost $30 \mathrm{~dB} \mu \mathrm{V}$ in the frequency range of interest.

However, the attenuation was not as spectacular as the catalog specification's sheet, which confirms once again our above allegation about the manufactures' specifications, generally optimistic compared with their performances in working equipments.

Practically, the troubleshooting of the computer's firmware package was greatly improved after the insertion of the EMI filter cascade at the port of the treadmill equipment.

\section{Conclusion and Further Works}

EMC is an important stage of system design today and will become more stringent as time moves on.

One must keep in mind that when switching occurs, so does noise whether it be conducted or radiated noise.

Once again, manufacturers of electronic products must realize that simply complying with the regulatory limits on conducted and radiated emissions does not represent a complete design, from the standpoint of EMC.

A product must be reasonably insensitive to disturbances that are present on the power system network in order to ensure reliable operation of the product.
Virtually all products contain a power supply filter as the last circuit that noise currents pass through before they exit the product through the power cord.

The decomposition of the total currents into commonmode and differential-mode components along with this realization that each element of the power supply filter affects one and only one of these components is the key to designing power supply filters that are effective in the reduction of conducted emissions so that the product will comply with the regulatory limits.

In the case presented in this paper we have a plenty of conducted interference paths, some of them being depicted in Fig. 1, the others being hidden interference paths, quite difficult to "catch".

However, all of them should be carefully analyzed in the terms of electromagnetic compatibility, either from the point of view of conducted emissions and in the same time from the point of view of radiated emissions. As we know, conducted emissions have a tremendous potential in generating radiations, intra or inter system.

For the time being we have obtained consistent results only at the mains AC port of the treadmill, for the point of view of conducted emissions in the standardized frequency range from $150 \mathrm{kHz}-30 \mathrm{MHz}$; our analysis revealed the necessity for retro fitting an extra EMI filter, provided with a proper common mode choke.

Generally speaking, in the case of switching equipments, mains filters are essential to meet regulations and, as we have seen, in certain conditions, it might be necessary to use an extra EMI filtering cell or to replace the existing filter.

We must also recall that the EMC problem must represent a continuous concern from the earliest moment of the design stage and the EMI filter issues have to be considered with very much attention. We always must keep in mind that in design activity, EMI filters shouldn't be considered only ancillary devices, as they are often treated, but a crucial matter in a successful operation.

\section{References}

[1] Williams, T., EMC for Product Designers, Newnes, 2007.

[2] Paul, C. R., Introduction to Electromagnetic Compatibility, Second Edition, John Wiley \& Sons Inc., 2006.

[3] Barnes, M., Practical Variable Speed Drives and Power Electronics, Newnes, 2003.

[4] Buzdugan, M. I., Bălan, H., Simion, E. E., Buzdugan, T. I., Electromagnetic interference at the mains ports of an equipment, ICREPQ 2008, Santander, 2008.

[5] Line Impedance Stabilization Network HM6050-2; Operating Manual; HAMEG Instruments.

[6] Spectrum analyzer HM 5014; Operating Manual; HAMEG Instruments.

[7] EN 55011: 2007 Industrial, scientific and medical (ISM) radio-frequency Equipment; Electromagnetic disturbance characteristics; Limits and methods of measurement.

[8] Ozenbaugh, R. L., EMI Filter Design, Marcel Dekker, Inc. 2001.

[9] Winder, S., Analog and Digital Filter Design, Elsevier Science, 2002.

[10] www.shoshin.co.jp

[11] www.arnoldmagnetics.com

[12] Electromagnetic Compatibility for Power Converters, Application Note 4145, Fairchild Semiconductor Corporation, 2004.

[13] www.arcotronics.ltd.uk 